\title{
Genomic Analyses of Bifidobacterium moukalabense Reveal Adaptations to Frugivore/Folivore Feeding Behavior
}

\author{
Takahiro Segawa ${ }^{1}\left(\mathbb{D}\right.$, Satoshi Fukuchi ${ }^{2}$, Dylan Bodington ${ }^{3}$, Sayaka Tsuchida ${ }^{4,5} \mathbb{D}$, \\ Pierre Philippe Mbehang Nguema ${ }^{6}$, Hiroshi Mori ${ }^{7}$ (D) and Kazunari Ushida ${ }^{4,5, *(D)}$ \\ 1 Center for Life Science Research, University of Yamanashi, 1110 Shimokato, Chuo, \\ Yamanashi 409-3898, Japan; tsegawa@yamanashi.ac.jp \\ 2 Faculty of Engineering, Maebashi Institute of Technology, Maebashi, Gunma 371-0816, Japan; \\ sfukuchi@maebashi-it.ac.jp \\ 3 Department of Biological Sciences, Tokyo Institute of Technology, Tokyo 152-8550, Japan; \\ bodington@gmail.com \\ 4 Chubu University Academy of Emerging Sciences, Kasugai, Aichi 487-8501, Japan; \\ s_tsuchida@isc.chubu.ac.jp \\ 5 Graduate School of Life and Environmental Sciences, Kyoto Prefectural University, Kyoto 606-8522, Japan \\ 6 Research Institute of Tropical Ecology, Libreville BP 13354, Gabon; mbehangnguema@yahoo.fr \\ 7 Center for Information Biology, National Institute of Genetics, 1111 Yata, Mishima, Shizuoka 411-8540, Japan; \\ hmori@nig.ac.jp \\ * Correspondence: k_ushida@isc.chubu.ac.jp; Tel.: +81-568-51-9520
}

Received: 29 December 2018; Accepted: 1 April 2019; Published: 4 April 2019

\begin{abstract}
Despite the essential role of Bifidobacterium in health-promoting gut bacteria in humans, little is known about their functions in wild animals, especially non-human primates. It is difficult to determine in vivo the function of Bifidobacterium in wild animals due to the limited accessibility of studying target animals in natural conditions. However, the genomic characteristics of Bifidobacterium obtained from the feces of wild animals can provide insight into their functionality in the gut. Here, we analyzed the whole genomes of $12 \mathrm{~B}$. moukalabense strains isolated from seven feces samples of wild western lowland gorillas (Gorilla gorilla gorilla), three samples of wild central chimpanzees (Pan troglodytes troglodytes) and two samples of wild forest elephants (Loxodonta cyclotis) in Moukalaba-Doudou National Park, Gabon. In addition, we analyzed the fecal bacterial communities of six wild western lowland gorillas by meta 16S rRNA gene analyses with next generation sequencing. Although the abundance of the genus Bifidobacterium was as low as $0.2 \%$ in the total reads, a whole genome analysis of $B$. moukalabense suggested its contribution digestion of food and nutrition of frugivore/folivore animals. Specifically, the whole genome analysis indicated the involvement of $B$. moukalabense in hemicellulose degradation for short chain fatty acid production and nucleic acid utilization as nitrogen resources. In comparison with human-associated Bifidobacterium spp., genes for carbohydrate transport and metabolism are not conserved in these wild species. In particular the glycosidases, which are found in all 12 strains of B. moukalabense, were variably detected, or not detected, in human-associated species.
\end{abstract}

Keywords: Bifidobacterium moukalabense; genomic characteristics; wild gorillas; wild chimpanzees; wild forest elephants

\section{Introduction}

The gut microbiome is composed of an immense diversity of microorganisms [1], and recent studies have shown their essential roles in host health [2-7]. Among these, the genus Bifidobacterium 
is the unique Actinobacteria, which is adapted to animal intestinal tracts. Bifidobacteria have been commonly recognized as health-promoting gut bacteria in many health-oriented research studies [8-12]. At present, the genus Bifidobacterium comprises 70 established species with 10 subspecies according to LPSN (http:/ / www.bacterio.net/bifidobacterium.html). Within these 70 species, human-associated species of Bifidobacterium are abundant. In contrast, other animal species often carry bifidobacteria with much lower levels of diversity [13]. In fact, the human gut normally harbors Bifidobacterium as one of the most abundant bacterial genera ( $10 \%)$ [14], and to date, 12 species belonging to $B$. adolescentis-group, B. longum-group or B. scardovi-group and B. bifidum have been recognized as human-associated bifidobacteria [15]. The wide phylogenetic variety of human-associated Bifidobacterium spp. suggests their adaptation to the wide range of eating habits of humans [16].

Several comparative genomic studies of Bifidobacterium have been conducted to assess the genomic characteristics of this particular genus in terms of adaptation to the human intestinal tract [17-19]. The discussion mostly focused on carbohydrate metabolism and adaptation to a wide variety of dietary carbohydrates. In addition, bile acid tolerance of Bifidobacterium spp. has been identified in some of the probiotic strains [20-22]. The wide variety of food carbohydrates and wide phylogenetic diversity of human-associated Bifidobacterium spp. may be related.

B. moukalabense was first isolated from a wild western lowland gorilla (Gorilla gorilla gorilla) [23]. According to the $16 \mathrm{~S}$ rRNA phylogeny, B. moukalabense belongs to the B. adolescentis-group and is closely related to $B$. catenulatum and $B$. pseudocatenulatum. The draft genome sequence reported later on this strain $\mathrm{GG} 01^{\mathrm{T}}$ indicated that this strain is close to $B$. dentium, the other member of the B. adolescentis group [24]. Genetic comparisons between B. moukalabense and those from human associated Bifidobacterium can address the question of why this species has not been identified from human microbiota, while it was identified in Homininae primates.

Since our first isolation of this particular B. moukalabense [23], we have successfully isolated several other strains of $B$. moukalabense from wild western lowland gorillas, wild central chimpanzees (Pan troglodytes troglodytes) and wild forest elephants (Loxodonta cyclotis) in the same forest in Moukalaba-Doudou National Park, Gabon. In this study, we carried out genomic analyses of 12 strains involving $\mathrm{GG} 01^{\mathrm{T}}$ to reveal the genomic characteristics by comparing the annotated gene functionality with those of human-associated Bifidobacterium species. The genomic differences and similarities between B. moukalabense and human-associated Bifidobacterium spp. suggested functional adaptations of B. moukalabense to the frugivore/folivore feeding behavior of the host.

\section{Materials and Methods}

\subsection{Isolation and Identification of Bifidobacteria}

Bifidobacteria were isolated from the feces of wild western lowland gorillas, central chimpanzees and forest elephants in Moukalaba-Doudou National Park in Gabon (Table 1). Seven gorilla feces samples and three chimpanzee feces samples were obtained by closely (10 to $15 \mathrm{~m}$ ) following a troop of gorilla or a troop of chimpanzee. Therefore, it was confirmed by visual observation that all feces were defecated by different individuals. Two elephant feces samples were obtained from a troop of three or four individuals. Since the wild forest elephants in this forest are not accustomed to human observers, it was difficult to follow them closely. Accordingly, it was not possible to conclude that these two elephant feces samples were defecated by different individuals, although the two feces samples were found within about $20 \mathrm{~m}$ distance from each other. The bifidobacterial strains used in this study were isolated and identified as described previously [23]. Briefly, loopful feces was applied to a Bifidobacterium-selective (BS) agar medium supplemented with $5 \%$ defibrinated horse blood at the sampling site. The plates were then quickly placed under anaerobic conditions in a pouch with an AnaeroPack (Mitsubishi Gas Chemical, Tokyo, Japan). After returning to the camp site, the pouches were placed in a styrene foam box with hand warmers, which heat the plates. The temperature was controlled manually in order to maintain storage, as far as this was possible, at $37^{\circ} \mathrm{C}$. After returning 
to the laboratory in Libreville, Gabon, the developed colonies were purified and the colony purity was confirmed microscopically. Seven, three and two isolates were respectively obtained from seven freshly defecated gorilla feces samples, three freshly defecated chimpanzee feces samples and two freshly defecated elephant feces samples. Isolates were grown for $24 \mathrm{~h}$ on the same plate medium, and developed colonies were subjected to DNA isolation using the previously described methods, with bead beating in a 5\% $(v / v)$ Triton X-100 [23].

PCR amplification of the 16S rRNA gene was performed using EX-Taq polymerase (Takara, Kyoto, Japan) and primers 27F and 1492R [25] with a thermal cycler (iCycler, BioRad, Tokyo, Japan). The PCR conditions were as follows: 3 min of initial denaturation at $95^{\circ} \mathrm{C}, 30$ cycles at $95^{\circ} \mathrm{C}$ for $30 \mathrm{~s}, 50^{\circ} \mathrm{C}$ for $30 \mathrm{~s}$ and $72{ }^{\circ} \mathrm{C}$ for $90 \mathrm{~s}$, and a final extension at $72{ }^{\circ} \mathrm{C}$ for $10 \mathrm{~min}$. The PCR products were purified using Wizard SV Gel and PCR Clean-Up System (Promega, Madison, WI, USA). Sequencing was achieved using Big Dye Terminator v3.1 and an ABI 3130xl automatic sequence analyzer (Applied Biosystems, Foster City, CA, USA). BLASTN searches were performed to identify the closest sequences in the NCBI-nt database.

\subsection{Genome Library Construction and Illumina Sequencing}

An aliquot of DNA (40-80 ng) from each isolate was sheared to a target peak size of $550 \mathrm{bp}$ using the Covaris S220 Focused-Ultrasonicator system (Covaris, Woburn, MA, USA) according to the manufacturer's recommendations. To generate DNA sequencing libraries for high-throughput DNA sequencing, a TruSeq DNA PCR-Free Library Preparation Kit (Illumina, San Diego, CA, USA) was used according to the manufacturer's instructions. The library products were isolated in agars gels (size of 500-800 bp) and purified by use of NucleoSpin Gel and PCR Clean-up (Takara, Japan). The sequencing library was used as a template for paired-end sequencing using a MiSeq Reagent Kit v3 (600 cyc) and the MiSeq desktop sequencer (Illumina, San Diego, CA, USA). Read files (fastq.gz) sequence reads were generated (average 927,054 read pairs per strain) using MiSeq Reporter software version 2.3.32 (Illumina, San Diego, CA, USA). 
Table 1. Bifidobacterium strains in this study.

\begin{tabular}{|c|c|c|c|c|c|c|c|}
\hline Bacterial Taxon & Strain Name & Source of Isolate & Common Name & $\begin{array}{l}\text { Location of } \\
\text { Collection }\end{array}$ & $\begin{array}{c}\text { Date of } \\
\text { Isolation }\end{array}$ & Collected by & Acession No. \\
\hline \multirow[t]{12}{*}{ Bifidobacterium moukalabense } & $\begin{array}{l}\text { GG01T (JCM18751, } \\
\text { DSM27321) }\end{array}$ & Gorilla gorilla gorilla & $\begin{array}{l}\text { Western Lowland } \\
\text { Gorilla }\end{array}$ & $\begin{array}{c}2^{\circ} 19^{\prime} 38^{\prime \prime} \mathrm{S} \\
10^{\circ} 34^{\prime} 02^{\prime \prime} \mathrm{E}\end{array}$ & $24 / 11 / 2010$ & $\begin{array}{l}\text { K. Ushida and } \\
\text { P.P.Nuguma }\end{array}$ & $\begin{array}{c}\text { PRJDB7909, } \\
\text { AZMV00000000 }\end{array}$ \\
\hline & GB01 & Gorilla gorilla gorilla & $\begin{array}{l}\text { Western Lowland } \\
\text { Gorilla }\end{array}$ & $\begin{array}{c}2^{\circ} 19^{\prime} 53^{\prime \prime} \mathrm{S} \\
10^{\circ} 34^{\prime} 06^{\prime \prime} \mathrm{E}\end{array}$ & $28 / 10 / 2009$ & $\begin{array}{l}\text { K. Ushida and } \\
\text { P.P.Nuguma }\end{array}$ & PRJDB7909 \\
\hline & GB03 & Gorilla gorilla gorilla & $\begin{array}{l}\text { Western Lowland } \\
\text { Gorilla }\end{array}$ & $\begin{array}{c}2^{\circ} 19^{\prime} 51^{\prime \prime} \mathrm{S} \\
10^{\circ} 34^{\prime} 19^{\prime \prime} \mathrm{E}\end{array}$ & $01 / 11 / 2009$ & $\begin{array}{l}\text { K. Ushida and } \\
\text { P.P.Nuguma }\end{array}$ & PRJDB7909 \\
\hline & GB04 & Gorilla gorilla gorilla & $\begin{array}{l}\text { Western Lowland } \\
\text { Gorilla }\end{array}$ & $\begin{array}{c}2^{\circ} 19^{\prime} 53^{\prime \prime} \mathrm{S} \\
10^{\circ} 34^{\prime} 21^{\prime \prime} \mathrm{E}\end{array}$ & $24 / 11 / 2010$ & $\begin{array}{l}\text { K. Ushida and } \\
\text { P.P.Nuguma }\end{array}$ & PRJDB7909 \\
\hline & GB62 & Gorilla gorilla gorilla & $\begin{array}{l}\text { Western Lowland } \\
\text { Gorilla }\end{array}$ & $\begin{array}{c}2^{\circ} 20^{\prime} 02^{\prime \prime} \mathrm{S} \\
10^{\circ} 34^{\prime} 05^{\prime \prime} \mathrm{E}\end{array}$ & $25 / 11 / 2010$ & $\begin{array}{l}\text { K. Ushida and } \\
\text { P.P.Nuguma }\end{array}$ & PRJDB7909 \\
\hline & GB63 & Gorilla gorilla gorilla & $\begin{array}{l}\text { Western Lowland } \\
\text { Gorilla }\end{array}$ & $\begin{array}{c}2^{\circ} 19^{\prime} 49^{\prime \prime} \mathrm{S} \\
10^{\circ} 34^{\prime} 10^{\prime \prime} \mathrm{E}\end{array}$ & $26 / 11 / 2010$ & $\begin{array}{l}\text { K. Ushida and } \\
\text { P.P.Nuguma }\end{array}$ & PRJDB7909 \\
\hline & GB65 & Gorilla gorilla gorilla & $\begin{array}{l}\text { Western Lowland } \\
\text { Gorilla }\end{array}$ & $\begin{array}{l}2^{\circ} 19^{\prime} 50^{\prime \prime} \mathrm{S} \\
10^{\circ} 34^{\prime} 05^{\prime \prime} \mathrm{E}\end{array}$ & $27 / 11 / 2010$ & $\begin{array}{l}\text { K. Ushida and } \\
\text { P.P.Nuguma }\end{array}$ & PRJDB7909 \\
\hline & CD14 & Pan troglodytes troglodytes & $\begin{array}{c}\text { Central } \\
\text { chimpanzee }\end{array}$ & $\begin{array}{c}2^{\circ} 23^{\prime} 08^{\prime \prime} \mathrm{S} \\
10^{\circ} 33^{\prime} 14^{\prime \prime} \mathrm{E}\end{array}$ & $29 / 11 / 2011$ & $\begin{array}{l}\text { S. Tsuchida and } \\
\text { P.P Nguema }\end{array}$ & PRJDB7909 \\
\hline & CD16 & Pan troglodytes troglodytes & $\begin{array}{c}\text { Central } \\
\text { chimpanzee }\end{array}$ & $\begin{array}{l}2^{\circ} 23^{\prime} 08^{\prime \prime} \mathrm{S} \\
10^{\circ} 33^{\prime} 14^{\prime \prime} \mathrm{E}\end{array}$ & $29 / 11 / 2011$ & $\begin{array}{l}\text { S. Tsuchida and } \\
\text { P.P Nguema }\end{array}$ & PRJDB7909 \\
\hline & CD33 & Pan troglodytes troglodytes & $\begin{array}{c}\text { Central } \\
\text { chimpanzee }\end{array}$ & $\begin{array}{c}2^{\circ} 23^{\prime} 08^{\prime \prime} \mathrm{S} \\
10^{\circ} 33^{\prime} 14^{\prime \prime} \mathrm{E}\end{array}$ & $29 / 11 / 2011$ & $\begin{array}{l}\text { S. Tsuchida and } \\
\text { P.P Nguema }\end{array}$ & PRJDB7909 \\
\hline & EB43 & Loxodonta cyclotis & $\begin{array}{c}\text { African forest } \\
\text { elephant }\end{array}$ & $\begin{array}{l}2^{\circ} 20^{\prime} 12^{\prime \prime} \mathrm{S} \\
10^{\circ} 34^{\prime} 31^{\prime \prime} \mathrm{E}\end{array}$ & $01 / 11 / 2009$ & $\begin{array}{l}\text { K. Ushida and } \\
\text { P.P.Nuguma }\end{array}$ & PRJDB7909 \\
\hline & EB44 & Loxodonta cyclotis & $\begin{array}{c}\text { African forest } \\
\text { elephant }\end{array}$ & $\begin{array}{l}2^{\circ} 20^{\prime} 12^{\prime \prime S} \\
10^{\circ} 34^{\prime} 31^{\prime \prime} \mathrm{E}\end{array}$ & $01 / 11 / 2009$ & $\begin{array}{l}\text { K. Ushida and } \\
\text { P.P.Nuguma }\end{array}$ & PRJDB7909 \\
\hline \multirow{7}{*}{$\begin{array}{c}\text { Bifidobacterium catenulatum } \\
\text { Bifidobacterium dentium } \\
\text { Bifidobacterium dentium } \\
\text { Bifidobacterium dentium } \\
\text { Bifidobacterium dentium } \\
\text { Bifidobacterium longum } \\
\text { Bifidobacterium } \\
\text { pseudocatenulatum }\end{array}$} & DSM 16992 & Homo sapiens & Human & & & & ID_BIFCAT_00411 \\
\hline & ATCC 27678 & Homo sapiens & Human & & & & ID_BIFDEN_01570 \\
\hline & ATCC 27679 & Homo sapiens & Human & & & & ID_HMPREF0168_0306 \\
\hline & Bd1 & Homo sapiens & Human & & & & ID_BDP_1602 \\
\hline & JCVIHMP022 & Homo sapiens & Human & & & & ID_HMPREF9003_0584 \\
\hline & DJO10A & Homo sapiens & Human & & & & ID_BLD_0124 \\
\hline & DSM 20438 & Homo sapiens & Human & & & & ID_BIFPSEUDO_03077 \\
\hline
\end{tabular}




\subsection{Phylogenetic Tree Construction Based on Core-Genome}

We discarded the Illumina MiSeq platform reads that contained ambiguous nucleotides, low qualily fastq reads (< average QV 25) and those mapped to the PhiX genome sequence. We also removed Illumina adapter sequences. These filtration steps were performed using Prinseq version 0.20.4 [26], Bowtie 2 version 2.1.0 [27] and Trimmomatic version 0.32 [28] with default parameters, respectively. Filtered sequencing reads were assembled into contigs using IDBA [29], a de novo assembler, with default parameters. Gene prediction and annotation was performed using Prokka [30], a tool for rapid annotation of prokaryotic genomes. The predicted genes from all sequenced strains and all reference strains were clustered into homologous gene families using get_homologues [31], which uses all-against-all BLASTP to define putative pairs of orthologs or recent paralogs. We compared the genomes of the B. moukalabense group from all isolates with human-associated Bifidobacterium, i.e., B. catenulatum, B. dentium, B. pseudocatenulatum, and B. longum, to reveal potentially adaptive function of Bifidobacterium in non-human frugivore/folivore animals. B. longum, which constitutes a neighboring phylogenetic group of the B. adolescentis group [15] was involved in this genomic analysis as a form of out-group. Clustering of genes was performed using orthoMCL [32] with a max E-value of $10^{-5}$ and a minimum coverage of $75 \%$ in the BLASTP alignments. The core genome was constructed using clusters of only single-copy genes from all sequenced and reference strains, and a pangenome matrix was constructed to manually inspect the non-core homologues. B. catenulatum was used as the reference for annotation for all homologous clusters in the core genome set, while clusters outside the core genome (B. dentium, B. pseudocatenulatum and B. longum) were manually curated by inspecting the annotations for all strains present in the cluster. The proteins were classified by the COG functional category [33]. The genomes studied are listed in Table S1. The core-genome DNA sequences were aligned using the program MAFFT and phylogenetic relationships among B. moukalabense, B. denitium, B. catenulatum and B. pseudocatenulatum were reconstructed based on their genomic data with the maximum likelihood method and the Bayesian method. The nucleotide substitution models were evaluated by the criterion of AIC, AICc, and BIC with the ModelFinder, and the GTR $+\mathrm{I}+\Gamma$ model was selected as the best model in all criterion. Maximum likelihood tree was reconstructed using RAxML version 8.2.10 [34] with the GTR $+\mathrm{I}+\Gamma$ model. To evaluate the confidences of the internal branches, the bootstrap method was applied by the rapid bootstrap algorithm [34] with 1000 replications. The Bayesian tree was reconstructed using the MrBayes version 3.2.6 with the GTR $+\mathrm{I}+\Gamma$ model under the two independent sets of the four simultaneous chains of the MCMC with the length of 100,000 generations. The trees and parameters were sampled every 100 generations. The first 10,000 generations were discarded as the burn-in. Carbohydrate-active enzymes were identified based on similarity to the carbohydrate-active enzyme (CAZy) database [35] using HMMER 3.1 software [36] with a max E-value cutoff of $1 \times 10^{-18}$.

\subsection{Orthologous Abundance Comparison of the Human Metagenome-Derived Bifidobacterium Genomes}

The 3973 draft Bifidobacterium genomes, derived from 9428 human metagenome samples, were obtained from a database (http:/ / opendata.lifebit.ai/table/SGB) [37]. Protein coding genes $(6,105,571$ genes) from the 3973 draft Bifidobacterium genomes were predicted using Prodigal version 2.6.3 with -p meta parameter [38]. The 5832 representative amino acid sequences of each orthologous cluster in the presently determined 12 genome data of $B$. moukalabense were searched for against the 6,105,571 amino acid sequences using MMseqs2 with -s 4 parameter [39], and filtered out with identity $<40 \%$ hits. The number of genomes which has homologous genes of each orthologous cluster was calculated.

\subsection{High Throughput Sequencing of $16 S$ rRNA Genes on Feces Samples}

Feces samples from gorillas were collected in August 2011 in Moukaraba-Doudou National Park, as indicated elsewhere [23]. Briefly, freshly defecated feces were collected from six adult gorillas and transferred in DNA conservation solution [40]. Partial 16S rRNA gene sequences including V3 
and V4 regions were amplified using primers Bakt_341F and Bakt_805R [41] with Illumina overhang adaptor sequences attached to their $5^{\prime}$ ends. $16 \mathrm{~S}$ rRNA gene amplicon libraries were constructed as described previously [42]. The amplicons with Illumina overhang adaptor sequences were triplicated and each amplicon was polled before index PCR using a Nextera XT Index kit (Illumina, San Diego, CA, USA). The resulting DNA was used as a template for paired-end sequencing using a MiSeq Reagent Kit v3 (600 cycles) and the MiSeq desktop sequencer. Genus compositions were inferred using VITCOMIC2 [43].

\subsection{Ethics}

The feces of wild gorillas, chimpanzees, and elephants were collected non-invasively, as indicated elsewhere [23]. The sampling was conducted with the collaboration of local veterinary staff within the SATREPS program (J. Yamagiwa, 2009-2014) under R/D DOCUMENTS between the Japanese International Cooperation Agency (JICA) and the Gabonese Government (Ministère de l'Enseignement Supérieur de la Recherche Scientifique et du Développement Technologique, Centre National de la Recherche Scientifique et Technologique and L'Institut de Recherche en Écologie Tropicale) and MOU between Kyoto University and L'Institut de Recherche en Écologie Tropicale. Permission of access to these wild animals and of DNA use was obtained from the Centre National de la Recherche Scientifique et Technologique as No. 0610/MENESRSI/CENAREST/CG/CST/CRI (26/10/2009), No.0743/MENESRSI/CENAREST/CG/CST/CRI (20/10/2010), No. 306/MENESRSI/CENAREST/ CG/CST/CRI (14/10/2011).

\section{Results}

The draft genome sequences of the 12 strains included 18 to 33 contigs with varying size up to 2.40-2.59 Mbps (Table S1) with relatively good N50 values (0.14 to $1.56 \mathrm{Mbp}$ ). Estimated genome sizes of 11 strains of $B$. moukalabense were $2.5 \mathrm{Mb} \pm 0.59$ (mean $\pm \mathrm{SD}$ ) with nearly $59.9 \%$ GC content. These genomes harbor from 1919 to 2143 coding sequences (CDSs), from 57 to 64 rRNAs, and 4 or 5 tRNAs. No plasmid associated genes were detected.

B. moukalabense was confirmed to be phylogenetically close to B. catenulatum and B. pseudocatenulatum and is a monophyletic species belonging to the $B$. adolescentis-group by $16 \mathrm{~S}$ rRNA gene analysis (Figure S1). However, the pangenome phylogenetic tree shows that $B$. moukalabense is close to B. dentium rather than B. catenulatum and B. pseudocatenulatum (Figure 1).

The total number of proteins in this study was 39,774 . They were organized into 5832 clusters, hereafter called "orthologous clusters". We categorized these proteins and orthologous clusters into seven levels according to the conservation level when compared with human-associated Bifidobacterium such as B. catenulatum, B. pseudocatenulatum, B. dentium, and B. longum (Tables S2 and S3). The frequencies of protein and orthologous clusters are shown in Figure S2. The remarkable feature in this analysis is that quite small numbers of proteins have been acquired in the human-associated species after divergence of these species and B. moukalabense (the numbers of proteins and orthologous clusters are 35 and 5 in level 6, see Figure S2). On the other hand, a considerable number of genes have been lost in the human-associated species as shown in level 1 and 2. In the comparison between 12 genomes of B. moukalabense (PRJDB7909) and 3973 meta-bifidobacterial genomes reconstructed from a human metagenome database [37], the conservation of genes was categorized into the 7 levels. The conservation pattern is shown in Figure 2, and is quite similar to those obtained by the comparison of 13 B. moukalabense genomes (PRJDB7909 and AZMV00000000) and seven human-associated Bifidobacterium. 


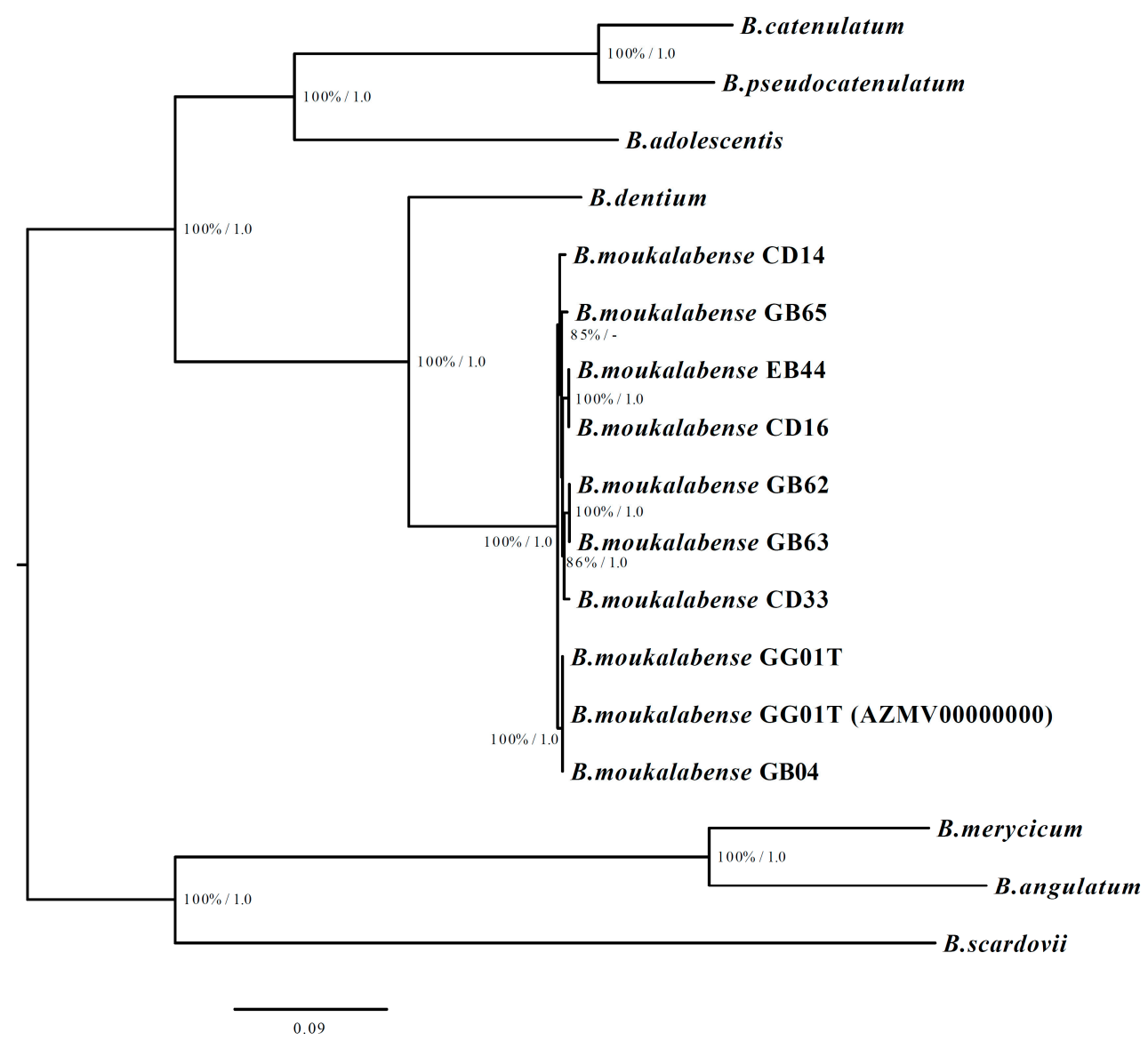

Figure 1. The maximum likelihood tree of B. moukalabense and its related species. The nodal numbers represent the bootstrap values (left) and Bayesian posterior probability (right). The branch lengths are proportional to the numbers of the nucleotide substitutions.

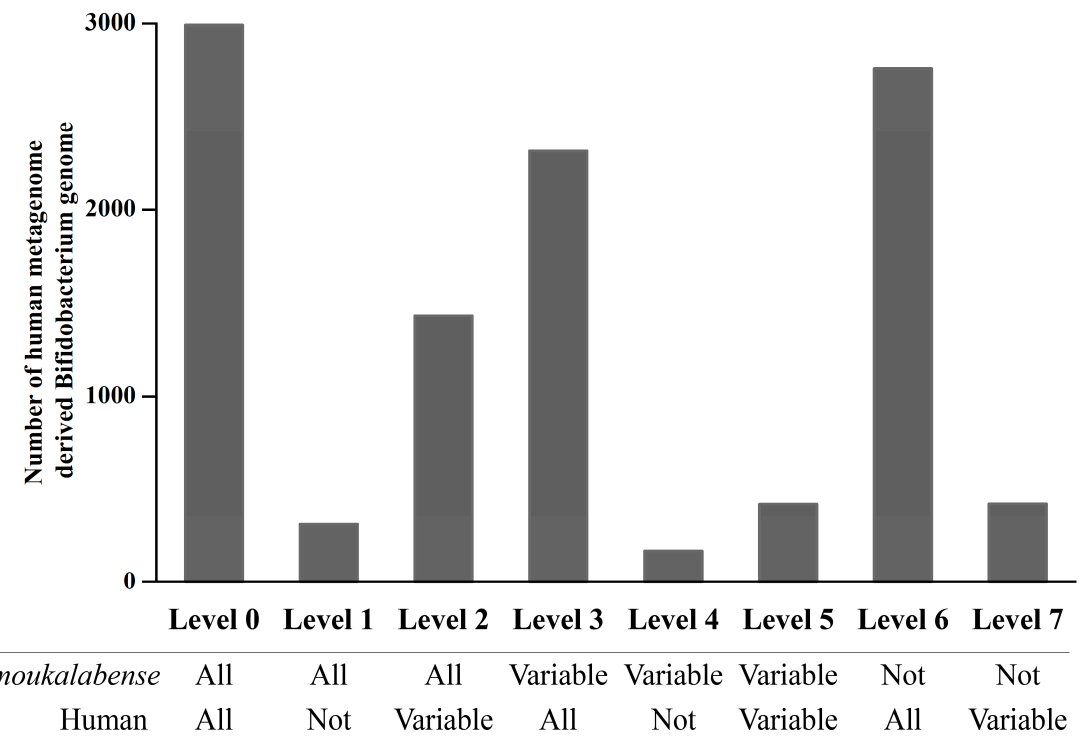

Figure 2. Numbers of shared orthologs derived from genomes of Bifidobacterium moukalabense and meta-bifidobacterial genomes reconstructed from human metagenome data. The $3973 \mathrm{draft}$ Bifidobacterium genomes were derived from 9428 human metagenome samples (37). Protein coding genes $(6,105,571$ genes) from the 3973 draft Bifidobacterium genomes were predicted. For details, see text. 
The conservation level, broken down by the COG category, is shown in Figure 3 and Table S4. The conservative functional categories are involved in essential biological activity, namely translation $(\mathrm{J})$, energy production $(\mathrm{C})$, amino acid, nucleotide, coenzyme, and lipid transport (E, F, H, and I respectively). On the other hand, the less conservative categories contain genes varying between B. moukalabense and human-associated species: defense mechanisms $(\mathrm{V})$, mobilome $(\mathrm{X})$, and secondary metabolites metabolism $(\mathrm{Q})$. The category of transcription $(\mathrm{K})$, cell wall and membrane $(\mathrm{M})$ and carbohydrate transport and metabolism $(\mathrm{G})$ are also the less conservative.

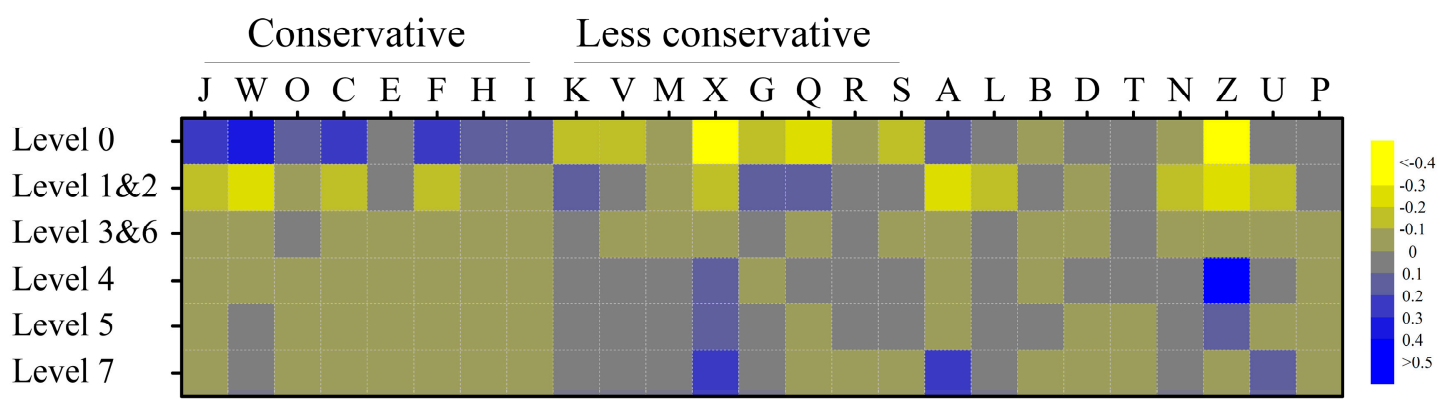

Figure 3. The frequency of the orthologs at different conservation level broken down by COG category.

When the genes were categorized by conservation levels between human-associated species and B. moukalabense, those in conservation levels 1 and 2 that are present in all B. moukalabense strains, and were variably present or not present in the human-associated species, were particularly over-represented in this functional category, carbohydrate transport and metabolism, showing that the genes involved in the carbohydrate metabolism are heterogeneous in the human-associated species (Tables S5-S8). Upon investigation of the gene content in category G, a remarkable feature is that the proportion of glycosidases in levels 1 and 2 was larger than the proportion of all of the other proteins in category G (Figure S3). The annotation of the orthologous cluster in category G and conservation level 2 is shown in Table S5. These glycosidases are mostly involved in the degradation of oligosaccharides to obtain energy from sugars.

Although the proteins associated with amino acid metabolism (E) and nucleotide metabolism (F) are conservative (Figure 3), a clear gene loss in the human-associated species is observed. We listed genes which are lost in human-associated species but which are present in all of the B. moukalabense strains (level 1 genes) in Table S6. In this table, a series of genes in a pathway associated with allantoin/hidantoin (orthologous cluster ID from 1792 to 1798) are lost in all human-associated species. As shown in Figure S4, these proteins are mapped on the pathway from allantoin to glycine in the KEGG pathway database [44].

Bacterial communities were also determined by meta 16S rRNA gene analysis with a Miseq platform for six feces samples collected from six wild western lowland gorillas. The abundance of the genus Bifidobacterium in fecal microbiota was calculated to be as low as $0.24 \% \pm 0.15$ (mean \pm SD) in wild western lowland gorillas (Table S9).

\section{Discussion}

In contrast to humans, Bifidobacterium is not recognized as the most prevalent bacterial group in non-human primates, particularly in wild individuals. In fact, only B. angulatum-like strains have been isolated from wild western chimpanzees (Pan troglodytes verus) in Bossou, Guinea and B. moukalabense is the sole Bifidobacterium isolated from wild western lowland gorillas [23,45]. This latter species was sole Bifidobacterium isolated from wild central chimpanzees in this study. However, only low levels ( $0.24 \%$ abundance) of the genus Bifidobacterium were recorded for six gorilla feces samples taken from the Moukalaba-Doudou National Park. Like wild western lowland gorillas, wild central chimpanzees showed low levels of Bifidobacterium in their gut microbiota [46]. It is noteworthy that B. moukalabense was also isolated from wild forest elephants in the same forest, although low levels of Bifidobacterium 
in their gut were reported [46,47]. Since forest elephants show similar feeding behavior to gorillas, and to a lesser extent, to chimpanzees [48-50], the sharing of the same species of Bifidobacterium by western lowland gorillas, central chimpanzees and forest elephants suggests that $B$. moukalabense is associated with frugivore/folivore feeding.

B. moukalabense belong to the B. adolescentis group which comprises B. dentium, B. catenulatum and B. pseudocatenulatum associated with the human gut. Captive-bred gorillas and chimpanzees harbored B. dentium, B. catenulatum and B. pseudocatenulatum instead of B. angulatum or B. moukalabense [51,52]. Any single species of the non-human Homininae in the natural condition is unlikely to host Bifidobacterium with such high density and divergency as in humans, as suggested by the present study (Table S9) and by previous studies [45-47,51]. An important point of our findings seems to be the fundamental differences in gene varieties, particularly in fiber degradation and nucleic acid metabolism between B. moukalabense and its phylogenetic neighbors, B. dentium, B. catenulatum and B. pseudocatenulatum. B. moukalabense is distinguished from B. dentium, B. catenulatum and B. pseudocatenulatum in the $B$. adolescentis group by the presence of a considerable number of unique genes. These unique genes may be important for the functional traits of B. moukalabense in the intestine of frugivore/folivores. The genome of B. moukalabense uniquely carries a range of carbohydrases (Tables S4 and S10, Figure S5); these carbohydrases are apparently homologs of those found in other intestinal bacteria such as Bacteroides thetaiotaomicron. Many of these genes are related to fiber degradation, namely hemicellulose degradation. Such a biased distribution of genes for hemicellulose degrading enzymes indicates that B. moukalabense may be related to the function of the frugivore/folivore food habit of its host.

Other strongly biased distributions of genes concerning nitrogen-scavenging capacity, such as nucleic acid catabolism, may also reflect the frugivore/folivore food habit of the host, in which the limitation of protein, i.e., amino acid, availability in the gut is expected (Table S1). Similar trends were observed in B. thermacidophilum genomes; domestic pig isolates apparently lost the genes for endoglucanase, beta-glucosidase, chitinase, an endoxylanase-related protein, beta-mannosidase, and pullulanase in comparison with wild boar isolates [53]. Instead, starch-related digestive enzymes such as maltodextrin glucosidase and alpha-glucosidase were detected only in pig isolates [53]. Wild boar isolates, thus, are apparently associated with fiber-rich omnivorous feeding behavior. In fact, cellulose and xylan comprise the major part of plant carbohydrates of the above-ground part, which comprises $70 \%$ of the plant materials consumed by wild boars [54]. Similarly, the unique presence of genes for fiber degradation may explain the association of $B$. moukalabense with a predicted fiber-rich intestinal environment found in wild boar isolates of $B$. thermacidophilum. The unique presence of genes for nucleic acid utilization in B. moukalabense explains its nitrogen scavenging ability under predicted protein- and amino acid-limited conditions in the intestinal environment. Our results are consistent with the nature of the diets of gorillas and elephants, and to a lesser extent, with that of chimpanzees, which are composed primarily of carbohydrates and are generally limited in protein [55]. The aforementioned differences in genomes between B. moukalabense with 7 known genomes of human-associated Bifidobacterium spp. were confirmed by a comparison between B. moukalabense and reconstructed meta bifidobacterial genomes. Since the meta-bifidobacterial genomes were reconstructed from huge data sets (9428 human metagenome samples), they can represent the human gut microbiome under a wide variety of feeding conditions.

In conclusion, B. moukalabense is a unique Bifidobacterium which is specialized to frugivore/folivore feeding habits of its host. In this context, this Bifidobacterium can reside in the gut of the typical herbivore animal like African forest elephants. But adaptation to frugivore/folivore feeding habits may not have been advantageous in omnivore human ancestors, who developed carnivore food habits [56].

Data Accessibility: The high-throughput sequencing data have been submitted to the DDBJ Sequence Read Archive under accession number PRJDB7909 and PRJDB7910. The B. moukalabense orthologous clusters in this study are available from http:/ / gorilla19.paleogenome.jp.

Supplementary Materials: The following are available online at http:/ /www.mdpi.com/2076-2607/7/4/99/s1, Figure S1: Neighbor joining phylogenetic tree of Bifidobacterium spp. based on 16S rRNA gene sequence; Figure S2: 
Frequency of the conservation levels of proteins and orthologous clusters; Figure S3: Frequency of the carbohydrate metabolism and transport (category G) proteins; Figure S4: Allantoin metabolism pathway in the KEGG map; Figure S5: Bray-Curtis dissimilarity based on the Carbohydrate Active Enzymes (CAZy) system; Table S1: Genome statistics of Bifidobacterium moukalabense in this study; Table S2: Conservation level of proteins and orthologous clusters into the 7 levels in this study; Table S3: COG category list; Table S4: The number of ortholog clusters and proteins of the conservation levels broken down into the COG functional categories; Table S5: The list of the level 2 ortholog clusters within COG category G (carbohydrate metabolism and transport); Table S6: The list of level 1 ortholog clusters with the COG annotation; Table S7: The list of level 2 ortholog clusters with the COG annotation; Table S8: The list of level 6 ortholog clusters with the COG annotation; Table S9: Ratio (\%) of fecal microbial community by $16 \mathrm{~S}$ rRNA gene analysis; Table S10: Classification according to the Carbohydrate Active Enzymes (CAZy) system.

Author Contributions: For research articles with several authors, a short paragraph specifying their individual contributions must be provided. The following statements should be used "conceptualization, K.U., S.T. and T.S.; methodology, T.S., S.F., D.B., S.T., P.P.M.N., H.M.; software, S.T., S.F., D.B., and H.M.; validation, T.S., and K.U.; formal analysis, T.S., S.F., D.B., H.M.; investigation, T.S., S.F., D.B., S.T., and H.M.; resources, S.T., P.P.M.N., and K.U.; data curation, T.S., S.F., D.B., H.M.; writing-original draft preparation, T.S., and K.U.; writing-review and editing, T.S., and K.U.; visualization, T.S., S.F., and S.T.; supervision, K.U.; project administration, K.U.; funding acquisition, S.T., and K.U.

Funding: This research was funded by the Japan Science and Technology Agency (JST) and Japan International Cooperation Agency (JICA), grant for the Science and Technology Research Partnership for Sustainable Development (SATREPS) "Conservation of Biodiversity in Tropical Forest through Sustainable Coexistence between Human and Wild Animals" (PI, Juichi Yamagiwa) and the study was also supported by Grants-in-Aid for Scientific Research (15K18775, Sayaka Tsuchida)".

Acknowledgments: We thank Ayumi Akiyoshi and Chiaki Hagiwara for technical assistance and Takahiro Yonezawa at Tokyo University of Agriculture for his valuable discussion about genetic evolution of Bifidobacterium. The authors are indebted for the sampling to Juichi Yamagiwa (Kyoto University), Yuji Takenoshita (Chubu Gakuin University), Shiho Fujita (Kagoshima University), Ludovic Ngok Banak and Alfred Ngomanda, the former and the actual Director of the Research Institute of Tropical Ecology (IRET)/National Center of Scientific Research and Technology (CENAREST) of Gabonese Republic. Takahiro Segawa was supported by Transdisciplinary Research Integration Center (TRIC) of the Research Organization of Information and Systems. Ortholog analyses were supported by Basis for Supporting Innovative Drug Discovery and Life Science Research program (2051). Computations were partially performed on the NIG (National Institute of Genetics) supercomputer at ROIS (Research Organization of Information and Systems) National Institute of Genetics.

Conflicts of Interest: The authors declare no conflicts of interest.

\section{References}

1. Arumugam, M.; Raes, J.; Pelletier, E.; Le Paslier, D.; Yamada, T.; Mende, D.R.; Fernandes, G.R.; Tap, J.; Bruls, T.; Batto, J.-M.; et al. Enterotypes of the human gut microbiome. Nature 2011, 473, 174-180. [CrossRef] [PubMed]

2. Anderson, J.L.; Edney, R.J.; Whelan, K. Systematic review: Faecal microbiota transplantation in the management of inflammatory bowel disease. Aliment. Pharmacol. Ther. 2012, 36, 503-516. [CrossRef]

3. Arpaia, N.; Campbell, C.; Fan, X.; Dikiy, S.; van der Veeken, J.; de Roos, P.; Liu, H.; Cross, J.R.; Pfeffer, K.; Coffer, P.J.; et al. Metabolites produced by commensal bacteria promote peripheral regulatory T-cell generation. Nature 2013, 504, 451-455. [CrossRef] [PubMed]

4. Le Chatelier, E.; Nielsen, T.; Qin, J.; Prifti, E.; Hildebrand, F.; Falony, G.; Almeida, M.; Arumugam, M.; Batto, J.-M.; Kennedy, S.; et al. Richness of human gut microbiome correlates with metabolic markers. Nature 2013, 500, 541-546. [CrossRef] [PubMed]

5. Belcheva, A.; Irrazabal, T.; Robertson, S.J.; Streutker, C.; Maughan, H.; Rubino, S.; Moriyama, E.H.; Copeland, J.K.; Surendra, A.; Kumar, S.; et al. Gut Microbial Metabolism Drives Transformation of Msh2-Deficient Colon Epithelial Cells. Cell 2014, 158, 288-299. [CrossRef]

6. Singh, N.; Gurav, A.; Sivaprakasam, S.; Brady, E.; Padia, R.; Shi, H.; Thangaraju, M.; Prasad, P.D.; Manicassamy, S.; Munn, D.H.; et al. Activation of Gpr109a, Receptor for Niacin and the Commensal Metabolite Butyrate, Suppresses Colonic Inflammation and Carcinogenesis. Immunity 2014, 40, 128-139. [CrossRef] [PubMed]

7. Smith, P.M.; Howitt, M.R.; Panikov, N.; Michaud, M.; Gallini, C.A.; Bohlooly-y, M.; Glickman, J.N.; Garrett, W.S. The Microbial Metabolites, Short-Chain Fatty Acids, Regulate Colonic T reg Cell Homeostasis. Science 2013, 341, 569-573. [CrossRef] 
8. Ashraf, R.; Shah, N.P. Immune System Stimulation by Probiotic Microorganisms. Crit. Rev. Food Sci. Nutr. 2014, 54, 938-956. [CrossRef]

9. Langkamp-Henken, B.; Rowe, C.C.; Ford, A.L.; Christman, M.C.; Nieves, C.; Khouri, L.; Specht, G.J.; Girard, S.-A.; Spaiser, S.J.; Dahl, W.J. Bifidobacterium bifidum R0071 results in a greater proportion of healthy days and a lower percentage of academically stressed students reporting a day of cold/flu: A randomised, double-blind, placebo-controlled study. Br. J. Nutr. 2015, 113, 426-434. [CrossRef] [PubMed]

10. Arboleya, S.; Watkins, C.; Stanton, C.; Ross, R.P. Gut Bifidobacteria Populations in Human Health and Aging. Front. Microbiol. 2016, 7, 1204. [CrossRef] [PubMed]

11. O'Callaghan, A.; van Sinderen, D. Bifidobacteria and Their Role as Members of the Human Gut Microbiota. Front. Microbiol. 2016, 7, 925. [CrossRef]

12. Turroni, F.; Berry, D.; Ventura, M. Editorial: Bifidobacteria and Their Role in the Human Gut Microbiota. Front. Microbiol. 2017, 7, 2148. [CrossRef]

13. Holzapfel, W.H.; Wood, B.J.B. Introduction to the LAB. In Lactic Acid Bacteria; John Wiley \& Sons, Ltd.: Hoboken, NJ, USA, 2014; pp. 1-12. [CrossRef]

14. Mitsuoka, T.; Kaneuchi, C. Ecology of the bifidobacteria. Am. J. Clin. Nutr. 1977, 30, 1799-1810. [CrossRef]

15. Bottacini, F.; Ventura, M.; van Sinderen, D.; O'Connell Motherway, M. Diversity, ecology and intestinal function of bifidobacteria. Microb. Cell Fact. 2014, 13, S4. [CrossRef]

16. Ventura, M.; Turroni, F.; van Sinderen, D. Bifidobacteria of the Human Gut: Our Special Friends A2-Tuohy, Kieran. In Diet-Microbe Interactions in the Gut; Rio, D.D., Ed.; Academic Press: San Diego, CA, USA, 2015; Chapter 4; pp. 41-51. [CrossRef]

17. Schell, M.A.; Karmirantzou, M.; Snel, B.; Vilanova, D.; Berger, B.; Pessi, G.; Zwahlen, M.-C.; Desiere, F.; Bork, P.; Delley, M.; et al. The genome sequence of Bifidobacterium longum reflects its adaptation to the human gastrointestinal tract. Proc. Natl. Acad. Sci. USA 2002, 99, 14422-14427. [CrossRef]

18. Sela, D.A.; Chapman, J.; Adeuya, A.; Kim, J.H.; Chen, F.; Whitehead, T.R.; Lapidus, A.; Rokhsar, D.S.; Lebrilla, C.B.; German, J.B.; et al. The genome sequence of Bifidobacterium longum subsp. infantis reveals adaptations for milk utilization within the infant microbiome. Proc. Natl. Acad. Sci. USA 2008, 105, 18964-18969.

19. Turroni, F.; Bottacini, F.; Foroni, E.; Mulder, I.; Kim, J.-H.; Zomer, A.; Sánchez, B.; Bidossi, A.; Ferrarini, A.; Giubellini, V.; et al. Genome analysis of Bifidobacterium bifidum PRL2010 reveals metabolic pathways for host-derived glycan foraging. Proc. Natl. Acad. Sci. USA 2010, 107, 19514-19519. [CrossRef]

20. Barrangou, R.; Briczinski, E.P.; Traeger, L.L.; Loquasto, J.R.; Richards, M.; Horvath, P.; Coûté-Monvoisin, A.-C.; Leyer, G.; Rendulic, S.; Steele, J.L.; et al. Comparison of the Complete Genome Sequences of Bifidobacterium animalis subsp. lactis DSM 10140 and Bl-04. J. Bacteriol. 2009, 191, 4144-4151. [CrossRef]

21. Andriantsoanirina, V.; Allano, S.; Butel, M.J.; Aires, J. Tolerance of Bifidobacterium human isolates to bile, acid and oxygen. Anaerobe 2013, 21, 39-42. [CrossRef]

22. Ruiz, L.; Margolles, A.; Sánchez, B. Bile resistance mechanisms in Lactobacillus and Bifidobacterium. Front. Microbiol. 2013, 4, 396. [CrossRef]

23. Tsuchida, S.; Takahashi, S.; Nguema, P.P.M.; Fujita, S.; Kitahara, M.; Yamagiwa, J.; Ngomanda, A.; Ohkuma, M.; Ushida, K. Bifidobacterium moukalabense sp. nov., isolated from the faeces of wild west lowland gorilla (Gorilla gorilla gorilla). Int. J. Syst. Evol. Microbiol. 2014, 64, 449-455. [CrossRef]

24. Lugli, G.A.; Duranti, S.; Milani, C.; Turroni, F.; Viappiani, A.; Mangifesta, M.; van Sinderen, D.; Ventura, M. The Genome Sequence of Bifidobacterium moukalabense DSM 27321 Highlights the Close Phylogenetic Relatedness with the Bifidobacterium dentium Taxon. Genome Announc. 2014, 2, e00048-00014. [CrossRef]

25. Kitahara, M.; Sakamoto, M.; Ike, M.; Sakata, S.; Benno, Y. Bacteroides plebeius sp. nov. and Bacteroides coprocola sp. nov., isolated from human faeces. Int. J. Syst. Evol. Microbiol. 2005, 55, 2143-2147. [CrossRef]

26. Edwards, R.; Schmieder, R. Quality control and preprocessing of metagenomic datasets. Bioinformatics 2011, $27,863-864$.

27. Langmead, B.; Salzberg, S.L. Fast gapped-read alignment with Bowtie 2. Nat. Methods 2012, 9, 357-359. [CrossRef]

28. Bolger, A.M.; Usadel, B.; Lohse, M. Trimmomatic: A flexible trimmer for Illumina sequence data. Bioinformatics 2014, 30, 2114-2120. [CrossRef]

29. Peng, Y.; Leung, H.C.M.; Yiu, S.M.; Chin, F.Y.L. IDBA-UD: A de novo assembler for single-cell and metagenomic sequencing data with highly uneven depth. Bioinformatics 2012, 28, 1420-1428. [CrossRef]

30. Seemann, T. Prokka: Rapid prokaryotic genome annotation. Bioinformatics 2014, 30, 2068-2069. [CrossRef] 
31. Contreras-Moreira, B.; Vinuesa, P. GET_HOMOLOGUES, a Versatile Software Package for Scalable and Robust Microbial Pangenome Analysis. Appl. Environ. Microbiol. 2013, 79, 7696-7701. [CrossRef]

32. Li, L.; Stoeckert, C.J.; Roos, D.S. OrthoMCL: Identification of Ortholog Groups for Eukaryotic Genomes. Genome Res. 2003, 13, 2178-2189. [CrossRef]

33. Tatusov, R.L.; Galperin, M.Y.; Natale, D.A.; Koonin, E.V. The COG database: A tool for genome-scale analysis of protein functions and evolution. Nucleic Acids Res. 2000, 28, 33-36. [CrossRef] [PubMed]

34. Stamatakis, A.; Hoover, P.; Rougemont, J. A rapid bootstrap algorithm for the RAxML web servers. Syst. Biol. 2008, 57, 758-771. [CrossRef] [PubMed]

35. Lombard, V.; Golaconda Ramulu, H.; Drula, E.; Coutinho, P.M.; Henrissat, B. The carbohydrate-active enzymes database (CAZy) in 2013. Nucleic Acids Res. 2014, 42, D490-D495. [CrossRef]

36. Finn, R.D.; Clements, J.; Eddy, S.R. HMMER web server: Interactive sequence similarity searching. Nucleic Acids Res. 2011, 39, W29-W37. [CrossRef]

37. Pasolli, E.; Asnicar, F.; Manara, S.; Zolfo, M.; Karcher, N.; Armanini, F.; Beghini, F.; Manghi, P.; Tett, A.; Ghensi, P.; et al. Extensive Unexplored Human Microbiome Diversity Revealed by Over 150,000 Genomes from Metagenomes Spanning Age, Geography, and Lifestyle. Cell 2019, 176, 649-662. [CrossRef]

38. Hyatt, D.; Chen, G.-L.; LoCascio, P.F.; Land, M.L.; Larimer, F.W.; Hauser, L.J.J.B.B. Prodigal: Prokaryotic gene recognition and translation initiation site identification. BMC Bioinform. 2010, 11, 119. [CrossRef]

39. Steinegger, M.; Söding, J. MMseqs2 enables sensitive protein sequence searching for the analysis of massive data sets. Nat. Biotechnol. 2017, 35, 1026-1028. [CrossRef]

40. Hayaishi, S.; Kawamoto, Y. Low genetic diversity and biased distribution of mitochondrial DNA haplotypes in the Japanese macaque (Macaca fuscata yakui) on Yakushima Island. Primates 2006, 47, 158-164. [CrossRef]

41. Herlemann, D.P.R.; Labrenz, M.; Jürgens, K.; Bertilsson, S.; Waniek, J.J.; Andersson, A.F. Transitions in bacterial communities along the $2000 \mathrm{~km}$ salinity gradient of the Baltic Sea. ISME J. 2011, 5, 1571-1579. [CrossRef]

42. Rathnayake, R.M.L.D.; Oshiki, M.; Ishii, S.; Segawa, T.; Satoh, H.; Okabe, S. Effects of dissolved oxygen and $\mathrm{pH}$ on nitrous oxide production rates in autotrophic partial nitrification granules. Bioresour. Technol. 2015, 197, 15-22. [CrossRef]

43. Mori, H.; Maruyama, T.; Yano, M.; Yamada, T.; Kurokawa, K.J.B.S.B. VITCOMIC2: Visualization tool for the phylogenetic composition of microbial communities based on 16S rRNA gene amplicons and metagenomic shotgun sequencing. BMC Syst. Biol. 2018, 12, 30. [CrossRef] [PubMed]

44. Kanehisa, M.; Furumichi, M.; Tanabe, M.; Sato, Y.; Morishima, K. KEGG: New perspectives on genomes, pathways, diseases and drugs. Nucleic Acids Res. 2017, 45, D353-D361. [CrossRef] [PubMed]

45. Ushida, K.; Uwatoko, Y.; Adachi, Y.; Soumah, A.G.; Matsuzawa, T. Isolation of Bifidobacteria from feces of chimpanzees in the wild. J. Gen. Appl. Microbiol. 2010, 56, 57-60. [CrossRef] [PubMed]

46. Tsuchida, S.; Ushida, K. Characterization of intestinal bacterial communities of western lowland gorillas Gorilla gorilla gorilla, central chimpanzees Pan troglodytes troglodytes, and a forest elephant Loxodonta africana cyclotis living in Moukalaba-Doudou National Park in Gabon. Tropics 2015, 23, 175-183. [CrossRef]

47. Muegge, B.D.; Kuczynski, J.; Knights, D.; Clemente, J.C.; González, A.; Fontana, L.; Henrissat, B.; Knight, R.; Gordon, J.I. Diet Drives Convergence in Gut Microbiome Functions Across Mammalian Phylogeny and Within Humans. Science 2011, 332, 970-974. [CrossRef] [PubMed]

48. Short, J. Diet and feeding behaviour of the forest elephant. Mammalia 1981, 45, 177-185. [CrossRef]

49. Yamagiwa, J.; Tsubokawa, K.; Inoue, E.; Ando, C.J.P. Sharing fruit of Treculia africana among western gorillas in the Moukalaba-Doudou National Park, Gabon: Preliminary report. Primates 2015, 56, 3-10. [CrossRef]

50. Wilfried, E.E.; Yamagiwa, J. Use of tool sets by chimpanzees for multiple purposes in Moukalaba-Doudou National Park, Gabon. Primates 2014, 55, 467-472. [CrossRef]

51. Ushida, K. Intestinal bacteria of chimpanzees in the wild and in captivity: An application of molecular ecological methodologies. In Primate Parasite Ecology "The Dynamics and Study of Host-Parasite Relationships"; Cambridge University Press: Cambridge, UK, 2009; pp. 283-295.

52. Uenishi, G.; Fujita, S.; Ohashi, G.; Kato, A.; Yamauchi, S.; Matsuzawa, T.; Ushida, K. Molecular analyses of the intestinal microbiota of chimpanzees in the wild and in captivity. Am. J. Primatol. 2007, 69, 367-376. [CrossRef] 
53. Tsuchida, S.; Maruyama, F.; Ogura, Y.; Toyoda, A.; Hayashi, T.; Okuma, M.; Ushida, K. Genomic Characteristics of Bifidobacterium thermacidophilum Pig Isolates and Wild Boar Isolates Reveal the Unique Presence of a Putative Mobile Genetic Element with tetW for Pig Farm Isolates. Front. Microbiol. 2017, 8, 1540. [CrossRef]

54. Ballari, S.A.; Barrios-García, M.N. A review of wild boar Sus scrofa diet and factors affecting food selection in native and introduced ranges. Mamm. Rev. 2014, 44, 124-134. [CrossRef]

55. Cheeke, P.R.; Dierenfeld, E.S. Comparative Animal Nutrition and Metabolism; Cambridge University Press: Cambridge, UK, 2010.

56. Mann, N. Meat in the human diet: An anthropological perspective. Nutr. Diet. 2007, 64 (Suppl. 4), S102-S107. [CrossRef]

(c) (

(C) 2019 by the authors. Licensee MDPI, Basel, Switzerland. This article is an open access article distributed under the terms and conditions of the Creative Commons Attribution (CC BY) license (http:/ / creativecommons.org/licenses/by/4.0/). 